\title{
A NOTE ON WEAK BEZOUT RINGS
}

R. E. WILLIAMS

A weak Bezout ring is defined in [1] as an integral domain with unit (not necessarily commutative) such that any two principal right ideals with nonzero intersection have sum and intersection which are again principal. In this note we weaken the condition that a ring be a weak Bezout ring by means of the following theorem which enables us to drop the condition that intersections be principal.

THEOREM. Let $K$ be an integral domain in which each two principal right ideals with nonzero intersection have a principal sum. Then $K$ is a weak Bezout ring.

The proof is an immediate consequence of the following

Lemma. Let $K$ be as in the above Theorem. If $a, b \in K$ and $a K+b K=K$, there is an invertible matrix in $K_{2}$ with first row $a, b$.

Proof. If $a=0$ or $b=0$, the proof is clear, so take $a \neq 0, b \neq 0$. Let $a x-b y=1$. We may always take $x \neq 0, y \neq 0$. Note that $a(x a-1)$ $=b y a$ and $b(y b+1)=a x b$ are in $a K \cap b K$. Consider the elements

$$
b_{1}=x a-1, \quad b_{2}=x b, \quad a_{1}=y a, \quad a_{2}=y b+1 .
$$

From $b_{2} y=b_{1} x, b_{1} K \cap b_{2} K \neq 0$, whence $b_{1} K+b_{2} K$ is principal, say $b_{1} K+b_{2} K=b_{3} K$. For some $c_{1}, c_{2}, r, s$ in $K$ we have

$$
b_{1}=b_{3} c_{1}, \quad b_{2}=b_{3} c_{2}, \quad b_{1} r+b_{2} s=b_{3} .
$$

Defining $a_{3}=a_{1} r+a_{2} s$ it follows that $a b_{3}=b a_{3}$, whence the above equations yield the relations

$$
b a_{1}=a b_{1}=a b_{3} c_{1}=b a_{3} c_{1}, \quad b a_{2}=a b_{2}=a b_{3} c_{2}=b a_{3} c_{2} .
$$

Cancelling $b$ in the appropriate equations, we obtain $a_{1}=a_{3} c_{1}$ and $a_{2}=a_{3} c_{2}$. Now this result and (2) may be inserted into (1) to obtain:

(4) $x a-b_{3} c_{1}=1, \quad x b-b_{3} c_{2}=0, \quad-y a+a_{3} c_{1}=0, \quad-y b+a_{3} c_{2}=1$.

In terms of matrices, (4) becomes

$$
\left[\begin{array}{rr}
x & -b_{3} \\
-y & a_{3}
\end{array}\right]\left[\begin{array}{ll}
a & b \\
c_{1} & c_{2}
\end{array}\right]=\left[\begin{array}{ll}
1 & 0 \\
0 & 1
\end{array}\right]
$$

As $a$ and $b$ have a nonzero common right multiple each matrix is invertible.

Received by the editors April 13, 1967. 
Proof of Theorem. Let $a_{0}, b_{0}$ be in $K$ with $a_{0} K+b_{0} K=d K \neq 0$. Then for some $a, b, x, y$ in $K, a_{0}=d a, b_{0}=d b, a x-b y=1$. By the Lemma, $a, b$ is the first row of an invertible matrix in $K_{2}$. Let the matrix and its inverse be as in (5) above. Now let $a k_{1}+b k_{2}=0$. By equating the elements of

$$
\left[\begin{array}{ll}
a & b \\
b_{3} & 0
\end{array}\right]\left[\begin{array}{l}
k_{1} \\
k_{2}
\end{array}\right]=\left[\left[\begin{array}{ll}
a & b \\
b_{3} & 0
\end{array}\right]\left[\begin{array}{rr}
x & -b_{3} \\
-y & a_{3}
\end{array}\right]\right]\left[\left[\begin{array}{ll}
a & b \\
c_{1} & c_{2}
\end{array}\right]\left[\begin{array}{l}
k_{1} \\
k_{2}
\end{array}\right]\right]
$$

and cancelling $b_{3}$ we obtain $k_{1}=-b_{3}\left(c_{1} k_{1}+c_{2} k_{2}\right)$. Thus $a K \cap b K=a b_{3} K$. It follows that $a_{0} K \cap b_{0} K=a_{0} b_{3} K$.

\section{REFERENCE}

1. P. M. Cohn, Noncommutative unique factorization domains, Trans. Amer. Math. Soc. 109 (1963), 452-464.

Kansas State University 